International Institute for Applied Systems Analysis - A-2361 Laxenburg - Austria Tel: +432236807 • Fax: +43223671313 • E-mail: info@iiasa.ac.at •Web: www.iiasa.ac.at

INTERIM REPORT IR-98-045/May

Evolutionary Branching

under Asymmetric Competition

Éva Kisdi(kisdi@ludens.elte.hu)

Approved by

Ulf Diec kmann (dieckman@iiasa.ac .at)

Project Coordinator, Adaptive Dynamics Network

Interim Reports on work of the International Institute for Applied Systems Analysis receive only limited review. Views or opinions expressed herein do not necessarily represent those of the Institute, its National Member Organizations, or other organizations supporting the work. 


\section{IIASA Studies In AdAPTIVE Dynamics No. 28}

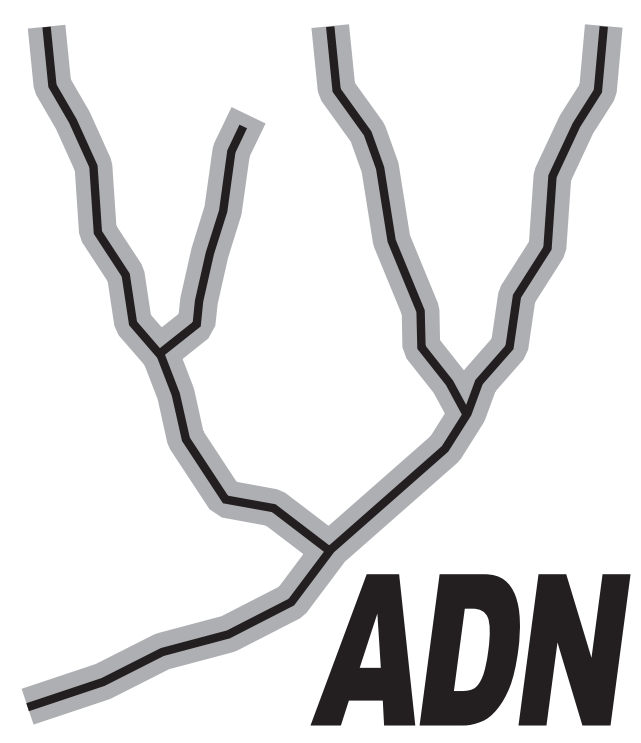

The Adaptive Dynamics Network at IIASA fosters the development of new mathematical and conceptual techniques for understanding the evolution of complex adaptive systems.

Focusing on these long-term implications of adaptive processes in systems of limited growth, the Adaptive Dynamics Network brings together scientists and institutions from around the world with IIASA acting as the central node.

Scientific progress within the network is reported in the IIASA Studies in Adaptive Dynamics series.

\section{The AdAPTIVe Dynamics NeTWORK}

The pivotal role of evolutionary theory in life sciences derives from its capability to provide causal explanations for phenomena that are highly improbable in the physicochemical sense. Yet, until recently, many facts in biology could not be accounted for in the light of evolution. Just as physicists for a long time ignored the presence of chaos, these phenomena were basically not perceived by biologists.

Two examples illustrate this assertion. Although Darwin's publication of "The Origin of Species" sparked off the whole evolutionary revolution, oddly enough, the population genetic framework underlying the modern synthesis holds no clues to speciation events. A second illustration is the more recently appreciated issue of jump increases in biological complexity that result from the aggregation of individuals into mutualistic wholes.

These and many more problems possess a common source: the interactions of individuals are bound to change the environments these individuals live in. By closing the feedback loop in the evolutionary explanation, a new mathematical theory of the evolution of complex adaptive systems arises. It is this general theoretical option that lies at the core of the emerging field of adaptive dynamics. In consequence a major promise of adaptive dynamics studies is to elucidate the long-term effects of the interactions between ecological and evolutionary processes.

A commitment to interfacing the theory with empirical applications is necessary both for validation and for management problems. For example, empirical evidence indicates that to control pests and diseases or to achieve sustainable harvesting of renewable resources evolutionary deliberation is already crucial on the time scale of two decades.

The Adaptive Dynamics Network has as its primary objective the development of mathematical tools for the analysis of adaptive systems inside and outside the biological realm. 


\section{IIASA StUdies in AdAPTIVe Dynamics}

No. 1 Metz JAJ, Geritz SAH, Meszéna G, Jacobs FJA, van Heerwaarden JS: Adaptive Dynamics: A Geometrical Study of the Consequences of Nearly Faithful Reproduction.

IIASA Working Paper WP-95-099.

van Strien SJ, Verduyn Lunel SM (eds.): Stochastic and Spatial Structures of Dynamical Systems, Proceedings of the Royal Dutch Academy of Science (KNAW Verhandelingen), North Holland, Amsterdam, pp. 183-231 (1996).

No. 2 Dieckmann U, Law R:

The Dynamical Theory of Coevolution: A Derivation from Stochastic Ecological Processes.

IIASA Working Paper WP-96-001.

Journal of Mathematical Biology (1996) 34, 579-612.

No. 3 Dieckmann U, Marrow P, Law R:

Evolutionary Cycling of Predator-Prey Interactions: Population Dynamics and the Red Queen.

Journal of Theoretical Biology (1995) 176, 91-102.

No. 4 Marrow P, Dieckmann U, Law R:

Evolutionary Dynamics of Predator-Prey Systems: An Ecological Perspective.

IIASA Working Paper WP-96-002.

Journal of Mathematical Biology (1996) 34, 556-578.

No. 5 Law R, Marrow P, Dieckmann U:

On Evolution under Asymmetric Competition.

IIASA Working Paper WP-96-003.

Evolutionary Ecology (1997) 11, 485-501.

No. 6 Metz JAJ, Mylius SD, Diekmann O:

When Does Evolution Optimise? On the Relation between Types of Density Dependence and Evolutionarily Stable Life History Parameters.

IIASA Working Paper WP-96-004.

No. 7 Ferrière R, Gatto M:

Lyapunov Exponents and the Mathematics of Invasion in Oscillatory or Chaotic Populations.

Theoretical Population Biology (1995) 48, 126-171.

No. 8 Ferrière R, Fox GA:

Chaos and Evolution.

Trends in Ecology and Evolution (1995) 10, 480-485.

No. 9 Ferrière R, Michod RE:

The Evolution of Cooperation in Spatially Heterogeneous Populations.

IIASA Working Paper WP-96-029.

American Naturalist (1996) 147, 692-717. 
No. 10 Van Dooren TJM, Metz JAJ:

Delayed Maturation in Temporally Structured Populations with Non-

Equilibrium Dynamics.

IIASA Working Paper WP-96-070.

Journal of Evolutionary Biology (1998) 11, 41-62.

No. 11 Geritz SAH, Metz JAJ, Kisdi E, Meszéna G:

The Dynamics of Adaptation and Evolutionary Branching.

IIASA Working Paper WP-96-077.

Physical Review Letters (1997) 78, 2024-2027.

No. 12 Geritz SAH, Kisdi E, Meszéna G, Metz JAJ:

Evolutionarily Singular Strategies and the Adaptive Growth and Branching of the Evolutionary Tree.

IIASA Working Paper WP-96-114.

Evolutionary Ecology (1998) 12, 35-57.

No. 13 Heino M, Metz JAJ, Kaitala V:

Evolution of Mixed Maturation Strategies in Semelparous Life-Histories:

the Crucial Role of Dimensionality of Feedback Environment.

IIASA Working Paper WP-96-126.

Philosophical Transactions of the Royal Society of London Series B (1997) 352, $1647-1655$.

No. 14 Dieckmann U:

Can Adaptive Dynamics Invade?

IIASA Working Paper WP-96-152.

Trends in Ecology and Evolution (1997) 12, 128-131.

No. 15 Meszéna G, Czibula I, Geritz SAH:

Adaptive Dynamics in a Two-Patch Environment: a Simple Model for Allopatric and Parapatric Speciation.

IIASA Interim Report IR-97-001.

Journal of Biological Systems (1997) 5, 265-284.

No. 16 Heino M, Metz JAJ, Kaitala V:

The Enigma of Frequency-Dependent Selection.

IIASA Interim Report IR-97-061.

Trends in Ecology and Evolution (1998) in press.

No. 17 Heino M:

Management of Evolving Fish Stocks.

IIASA Interim Report IR-97-062.

Canadian Journal of Fisheries and Aquatic Sciences (1998) in press.

No. 18 Heino M:

Evolution of Mixed Reproductive Strategies in Simple Life-History Models.

IIASA Interim Report IR-97-063.

No. 19 Geritz SAH, van der Meijden E, Metz JAJ:

Evolutionary Dynamics of Seed Size and Seedling Competitive Ability.

IIASA Interim Report IR-97-071.

No. 20 Galis F, Metz JAJ:

Why are there so many Cichlid Species? On the Interplay of Speciation and Adaptive Radiation.

IIASA Interim Report IR-97-072.

Trends in Ecology and Evolution (1998) 13, 1-2. 
No. 21 Boerlijst MC, Nowak MA, Sigmund K:

Equal Pay for all Prisoners. / The Logic of Contrition.

IIASA Interim Report IR-97-073.

AMS Monthly (1997) 104, 303-307.

Journal of Theoretical Biology (1997) 185, 281-294.

No. 22 Law R, Dieckmann U:

Symbiosis without Mutualism and the Merger of Lineages in Evolution.

IIASA Interim Report IR-97-074.

Proceedings of the Royal Society of London Series B (1998) 265, 1245-1253.

No. 23 Klinkhamer PGL, de Jong TJ, Metz JAJ:

Sex and Size in Cosexual Plants.

IIASA Interim Report IR-97-078.

Trends in Ecology and Evolution (1997) 12, 260-265.

No. 24 Fontana W, Schuster P:

Shaping Space: The Possible and the Attainable in RNA Genotype-

Phenotype Mapping.

IIASA Interim Report IR-98-004.

No. 25 Kisdi E, Geritz SAH:

Adaptive Dynamics in Allele Space: Evolution of Genetic Polymorphism by Small Mutations in a Heterogeneous Environment.

IIASA Interim Report IR-98-038.

No. 26 Fontana W, Schuster P:

Continuity in Evolution: On the Nature of Transitions.

IIASA Interim Report IR-98-039.

Science (1998) 280, 1451-1455.

No. 27 Nowak MA, Sigmund K:

Evolution of Indirect Reciprocity by Image Scoring. / The Dynamics of

Indirect Reciprocity.

IIASA Interim Report IR-98-040.

Nature (1998) 393, 573-577.

No. 28 Kisdi E:

Evolutionary Branching Under Asymmetric Competition.

IIASA Interim Report IR-98-045.

Issues of the IIASA Studies in Adaptive Dynamics series can be obtained free of charge. Please contact:

Adaptive Dynamics Network

International Institute for Applied Systems Analysis

Schloßplatz 1

A-2361 Laxenburg

Austria

Telephone +432236 807, Telefax +43 2236 71313, E-Mail adn@iiasa.ac.at, Internet http://www.iiasa.ac.at/Research/ADN 


\section{Contents}

Introduction $\quad 1$

The Model 3

Adaptive Dynamics under Asymmetric Competition 4

Examples $\quad 8$

Example 1: Linear $\rho(x) \quad 8$

$\begin{array}{lr}\text { Example 2: Gaussian } \rho(x) & 10\end{array}$

$\begin{array}{ll}\text { Example 3: Convex } \rho(x) & 12\end{array}$

$\begin{array}{ll}\text { Discussion } & 14\end{array}$

$\begin{array}{ll}\text { References } & 19\end{array}$ 


\begin{abstract}
I investigate the evolution of a continuous trait, such as body size or arms level, which affects the outcome of competitive contests such that the contestant with the larger trait value has a higher probability of winning. I show that a polymorphism of distinctly different strategies can evolve in an initially monomorphic population even if mutations have only small phenotypic effect. In a simple Lotka-Volterra type model of asymmetric competition, I derive the conditions under which two strategies can gradually evolve from a single ancestral strategy; the evolution of higher level polymorphisms is studied by numerical analysis and computer simulations of specific examples. High levels of polymorphism may build up during evolution. The coevolution of strategies in polymorphic populations, however, may also lead to extinction, which decreases the level of polymorphism. I discuss whether the evolution of several haploid strategies from a single initial strategy may correspond to the evolution of several sympatric species in a diploid outbreeding population.
\end{abstract}

Keywords: adaptive dynamics, asymmetric competition, coevolution, ESS, evolutionary Branching, frequency dependent selection 


\title{
About the Author
}

\author{
Éva Kisdi \\ Department of Genetics, Eötvös University \\ 1088 Budapest, Múzeum krt. 4/A, Hungary \\ and \\ Department of Zoology, University of Maryland, \\ College Park, MD 20742, USA
}

\section{Address for correspondence:}

Until 31 July, 1998:

Éva Kisdi, Adaptive Dynamics Network, International Institute for Applied Systems Analysis; e-mail: kisdi@iiasa.ac.at.

After 15 August, 1998:

Éva Kisdi, Department of Mathematics, University of Turku, FIN-20500 Turku, Finland, Fax: 358-21-633- 6595; e-mail any time: kisdi@ludens.elte.hu.

\section{Acknowledgments}

I thank Stefan Geritz and Peter Abrams for valuable discussions. This work was supported by the Hungarian National Scientific Research Foundation (OTKA, grant number T 019272), and by a travel grant from the Soros Foundation (Hungary). 


\title{
Evolutionary Branching Under Asymmetric Competition
}

\author{
Éva Kisdi
}

\section{Introduction}

The outcome of competitive interactions often depend on traits which influence or indicate competitive ability, such as body size, weight, armament, or costly signals of strength (e.g., Clutton-Brock et al., 1979; Clutton-Brock and Albon, 1979; Weiner, 1986, 1990; Weiner and Thomas, 1986; Carroll and Salamon, 1995; Luiselli, 1996; Mitani et al., 1996; Roberts, 1996; Simmons and Scheepers, 1996). The contestant with the larger trait value has a higher probability of winning the contest; large values of the trait, however, are costly in terms of reduced survival probability or reduced fecundity. Competitive asymmetry between members of a population has been recognized as an important factor for example in shaping within-population variability (Begon, 1984; Begon et al., 1996) and in stabilizing population dynamics (Lomnicki, 1989). Asymmetry is also prevalent in between-species competition (Lawton and Hassell, 1981; Connell, 1983; Schoener, 1983; Englund et al., 1992;), where the larger species is usually superior in interference competition (Persson, 1985; Alatalo and Moreno, 1987; Dickman, 1988; Thompson and Fox, 1993).

A wide range of evolutionary dynamics may occur for traits which determine competitive success. It is intuitively appealing that the competitive advantage of strategies which are larger than the rest of the population may lead to runaway evolution or an 'ams race. Dawkins and Krebs, 1979; Maynard Smith and Brown, 1986). The evolutionary increase of the trait value, however, may come to a halt either because the cost of large trait values increases and eventually outweighs the advantage, or because population size decreases such that competition is relaxed (Parker, 1983; Abrams and Matsuda, 1994). The population may reach a unique stable strategy, but multiple attractors are also possible (Abrams and Matsuda, 1994). Evolution may lead to extinction either because the trait value increases without limit and the associated population density asymptotically decreases to zero, or because the evolutionarily stable population has such a low density that it is endangered by demographic or environmental stochasticity (Matsuda and Abrams, 1994). 
A population of large individuals may be invaded by considerably smaller mutants, which cannot win competitive contests against the residents, but which are free from the costs of large size. Invasion of small mutants may give rise to a stable dimorphism (Abrams and Matsuda, 1994), or to evolutionary cycles in which runaway evolution towards large trait values is reset when small mutants take over the population (Maynard Smith and Brown, 1986). Asymmetric competition can also maintain a continuous evolutionarily stable distribution of trait values (Maynard Smith and Brown, 1986; Geritz, 1995).

A series of coevolutionary models assumed that size differences not only influence competitive superiority but also imply niche differentiation (Roughgarden, 1979; Rummel and Roughgarden, 1985; Brown and Vincent, 1987; Taper and Case, 1992). These models yield either a stable coalition of several species, or taxon cycles initiated by the invasion of a new species, followed by directional coevolution, extinction, and reconstitution of the initial species assemblage. Coevolution of two species with different within- and between-species competition was modelled by Law et al. (1997), who found multiple evolutionary attractors, parallel evolution or character convergence, evolutionary cycles, and extinction of one species.

In this paper, I investigate the evolution of polymorphism under asymmetric competition. Previous models with evolutionarily stable polymorphic populations or multispecies coalitions either did not consider the dynamics of evolution leading to polymorhism, or assumed that invaders had the best strategy given the present composition of the resident population. Though the latter procedure is apt to identify evolutionarily stable coalitions, it may be unrealistic for the actual dynamics of evolution (Taper and Case (1992) discuss this assumption in more detail). Here I assume that mutants are phenotypically similar to the resident strategies already present, and investigate how polymorphisms of distinctly different strategies can evolve by small mutational steps.

In most cases when a slightly different mutant appears in a resident population, it either invades and replaces the former resident strategy, or dies out; repeated invasions result in directional evolution of the trait (Eshel, 1983; Taylor, 1989). The resident and the mutant can coexist only when directional evolution ceases, i.e., near a so-called evolutionarily singular point. The evolutionary singularity where directional evolution arrives at may be an ESS; it also may, however, lack evolutionary stability. Near a singularity of the latter type, the invading mutant can coexist with the former resident; moreover, the two strategies undergo divergent coevolution, which gives rise to two phenotypically distinct strategies (Metz et al., 1992, 1996; Eshel et al., 1997; Geritz et al., 1997, 1998). The emergence of a dimorphism in an initially monomorphic population (or an $n+1$-morphism in an $n$-morphic population) followed by the gradual differentiation of the two initially similar strategies is called evolutionary branching, and the singular point at which the process initiates a branching point (Metz et al., 1996; Geritz et al., 1997, 1998). 
Assuming small mutations, the level of polymorphism can increase only by evolutionary branching. Hence I explore the evolution of polymorphism under asymmetric competition primarily by searching for evolutionary branching points. However, as I will also demonstrate, the coevolution of strategies which have arisen by evolutionary branching can lead to the extinction of a strategy later, thus the population can fall back to a lower level of polymorphism again.

The specific model I assume is a Lotka-Volterra competition model, where the competitive coefficients are determined by the differences between the trait values of the contestants. First I investigate under which conditions a monomorphic population can undergo evolutionary branching and thus become dimorphic. This part of the model analysis can be done without restricting generality by assuming specific functional forms in the model. Second, I explore the evolutionary dynamics of polymorphic populations. Since this is possible only by numerical analysis, I give three examples with different functions assumed. The examples illustrate repeated evolutionary branching leading to high levels of polymorphism, evolutionarily stable polymorphisms, and extinction.

\section{The Model}

I investigate the evolution of a single continuous trait such as body size or arms level. The trait has two effects on fitness. First, individuals of the population engage in competitive interactions, where a trait value larger than that of the contestant confers an advantage. Asymmetric competition thus exerts frequency-dependent selection for larger trait values. Second, large values of the trait imply a cost in terms of low survival and/or fecundity, irrespectively of other individuals. Frequency-independent selection may be either stabilizing (both small and large trait values are disadvantageous, intermediate trait values are favoured) or directional (large trait values are costly, small trait values are favoured). For example, stabilizing selection is likely to operate on body size. Armaments used solely to gain competitive advantage monotonously decrease fitness in a competition-free environment.

Let $n$ denote the number of strategies present in the population, with trait values $x_{1}, \ldots, x_{n}$ and population densities $N_{l}, \ldots, N_{n}$. Population dynamics are described by the Lotka-Volterra equations,

$$
\frac{d N_{i}}{d t}=N_{i}\left[\rho\left(x_{i}\right)-\sum_{j=1}^{n} \alpha\left(x_{i}-x_{j}\right) N_{j}\right]
$$

where $\rho\left(x_{i}\right)$ is the intrinsic growth rate of strategy $x_{i}$ in a competition-free environment, and $\alpha\left(x_{i}-x_{j}\right)$ is the competition coefficient which describes the effect of strategy $x_{j}$ on strategy $x_{i} \cdot \rho(\mathrm{x})$ is a decreasing function at least for large values of $x$. The competitive coefficient, $\alpha\left(x_{i}-x_{j}\right)$, is a decreasing function of the difference between the trait value of the individual and that of its contestant: Individuals with large trait value experience 
little competition by small individuals, while small individuals suffer from high competition by large individuals. The concave-convex function

$$
\alpha\left(x_{i}-x_{j}\right)=c\left(1-\frac{1}{1+v \exp \left(-k\left(x_{i}-x_{j}\right)\right)}\right)
$$

shown in Fig. 1 is a flexible way of modeling this situation.

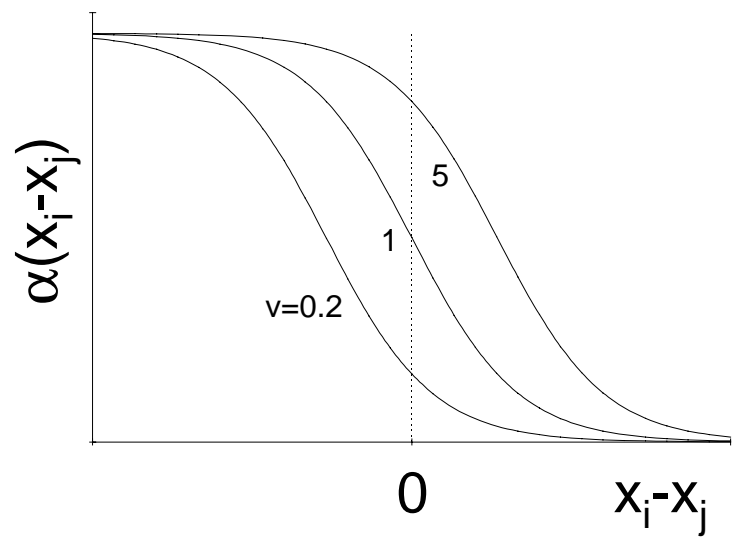

Fig. 1. Concave-convex shape of $\alpha$ as given by Eq. $2(v=0.2 ; 1 ; 5 ; c=2 ; k=4)$.

This model is similar to the one investigated recently by Law et al. (1997), with the following differences: (i) they considered two species with a single resident strategy for each; (ii) the intrinsic growth rate function $(\rho(\mathrm{x}))$ was assumed to be linear, and the competition coefficients were given by Eq. 2 with $v=1$; and (iii) different intensities of interspecific and intraspecific asymmetric competition (i.e., different values of $k$ and $c$ in Eq. 2 for within- and between-species competition) were allowed for. Here I consider an arbitrary number of coexisting strategies in a population, and assume that competition is determined solely by the trait values of the contestants. Except in the examples, I do not assume any particular function for $\rho(x)$ or $\alpha\left(x_{I}-x_{j}\right)$.

In order to analyze the dynamics of evolution, I assume that mutations are of small phenotypic effect. Evolution is mutation-limited, i.e., mutations occur infrequently such that a mutant strategy either has spread or has been excluded, and the population has reached its equilibrium by the time the next mutant comes along.

\section{Adaptive Dynamics under Asymmetric Competition}

In this section I start with a population of a single resident strategy, and investigate under which conditions this strategy can undergo evolutionary branching giving rise to two resident strategies. At the end of this section I briefly describe the generalized analysis for polymorphic resident populations.

Consider a rare mutant strategy $y$ in the resident population of strategy $x$. The mutant increases in number if its growth rate, 


$$
r_{x}(y)=\rho(y)-\alpha(y-x) N(x)
$$

is positive (cf. Eq. 1); a mutant with negative growth rate dies out. The resident population has zero growth rate $\left(r_{x}(x)=0\right)$ at the equilibrium population density $N(x)=\rho(x) / \alpha(0)$. It follows that a mutant strategy $y$ slightly larger than $x$ can invade and replace the resident if the fitness gradient

$$
\left.\frac{\partial r_{x}(y)}{\partial y}\right|_{y=x}=\rho^{\prime}(x)-\alpha^{\prime}(0) \rho(x) / \alpha(0)
$$

is positive; smaller mutants can invade if the fitness gradient is negative. Notice that since the resident and its slightly different mutant take part in competition, the shape of function $\alpha(y-x)$ is relevant only near $y-x=0$.

Repeated invasions and substitutions result in directional evolution until the population reaches an evolutionary singularity, where the fitness gradient is zero. At the singular strategy $x^{*}$, the proportionate cost of increasing the trait, $\left|\rho^{\prime}\left(x^{*}\right) / \rho\left(x^{*}\right)\right|$, must balance the constant advantage given by $\left|\alpha^{\prime}(0) / \alpha(0)\right|$ (cf. Eq. 4). If the cost is too high for all trait values, then frequency-independent selection drives evolution towards decreasing trait values despite the competitive advantage of being larger. Runaway evolution leads to increasing trait values if the proportionate cost is always smaller than the advantage for all trait values. Notice, however, that if $\rho(x)$ becomes zero at some $x_{\max }$, then the proportionate cost must exceed the advantage as $x$ increases, and therefore evolution must reach a singularity before $x_{\max }$.

Whether or not the population can undergo evolutionary branching at a singularity depends on two stability criteria (Metz et al., 1996; Geritz et al., 1997, 1998; Eshel et al., 1997). First, the singular strategy must be convergence stable such that directional evolution of a monomorphic population can approach it. This condition is fulfilled if

$$
\frac{d}{d x}\left[\left.\frac{\partial r_{x}(y)}{\partial y}\right|_{y=x}\right]_{x^{*}}=\left[\frac{\partial^{2} r}{\partial x \partial y}+\frac{\partial^{2} r}{\partial y^{2}}\right]_{y=x=x^{*}}<0
$$

(Eshel, 1983; Taylor, 1989; Christiansen, 1991). Second, the singularity must lack evolutionary stability, i.e.,

$$
\left[\frac{\partial^{2} r_{x}(y)}{\partial y^{2}}\right]_{y=x=x^{*}}>0
$$

(Maynard Smith, 1982). Substituting $r_{x}(y)$ from Eq. 3 into Inequalities 5 and 6, a singular strategy is a branching point if 


$$
\rho^{\prime \prime}\left(x^{*}\right)-\frac{\alpha^{\prime}(0)}{\alpha(0)} \rho^{\prime}\left(x^{*}\right)<0
$$

and

$$
\rho^{\prime \prime}\left(x^{*}\right)-\alpha^{\prime \prime}(0) \frac{\rho\left(x^{*}\right)}{\alpha(0)}>0
$$

The singularities that are both convergence stable and evolutionarily stable (i.e., satisfy Inequality 7 and the opposite of Inequality 8) are final stops of evolution; these were called continuously stable strategies or CSSs by Eshel (1983). Convergence unstable singularities (that do not satisfy Inequality 7) are evolutionary repellors.

The first derivatives in Inequality 7 are both negative: $\alpha^{\prime}(0)$ is negative by definition, and $\rho^{\prime}\left(x^{*}\right)$ must be negative otherwise the fitness gradient (Eq. 4) cannot be zero at $x *$. It follows from Inequality 7 that the singularity is always convergence stable if $\rho(x)$ is concave or linear, and may be convergence stable if $\rho(x)$ is convex. As seen from Inequality 8, convexity of $\rho(x)$ and a locally concave shape of $\alpha(y-x)$ near $y-x=0$ promote evolutionary branching. Table 1 summarizes the possible outcomes of evolution for different shapes of $\rho$ and $\alpha$.

Table 1. Possible evolutionary singularities with different functional forms for $\rho$ and $\alpha$.

\begin{tabular}{c|ccc}
\hline & \multicolumn{3}{|c}{$\alpha$ near zero } \\
& concave & $\begin{array}{c}\text { linear or } \\
\text { inflection } \\
\left(\alpha^{\prime \prime}(0)<0\right)\end{array}$ & convex \\
& $\begin{array}{c}\text { CSS or } \\
\text { concave } \rho\end{array}$ & CSS 0$)$ & $\left(\alpha^{\prime \prime}(0) \Rightarrow 0\right)$ \\
$\left(\rho^{\prime \prime}\left(x^{*}\right)<0\right)$ & branching point & & CSS \\
\hline linear $\rho$ & branching point & degenerate & CSS \\
$\left(\rho^{\prime \prime}\left(x^{*}\right)=0\right)$ & & & \\
\hline convex $\rho$ & branching point & branching point & CSS, branching \\
$\left(\rho^{\prime \prime}\left(x^{*}\right)>0\right)$ & or repellor & or repellor & point or repellor \\
\hline
\end{tabular}

If $\alpha^{\prime \prime}(0)=0$ and $\rho(x)$ is linear, then the monomorphic singular strategy is degenerate in the sense that $\left.\frac{\partial^{2} r_{x}(y)}{\partial y^{2}}\right|_{y=x=x^{*}}=0$, i.e., the singularity just undergoes a bifurcation between an ESS and a branching point. Since both the first and the second 
derivatives of the fitness function vanish at the singularity, it is the third derivative $\left.\frac{\partial^{3} r_{x}(y)}{\partial y^{3}}\right|_{y=x=x^{*}}$ that determines which mutants can invade. If the third derivative is positive, then mutants smaller than $x^{*}$ cannot invade the singular strategy, whereas a larger mutant can invade and coexist with $x^{*}$. A negative third derivative leads to the opposite result. The details of evolutionary dynamics in dimorphic populations near such a singularity are quite complicated, and will be presented elsewhere (Kisdi, in prep.). Ultimately, however, the population undergoes evolutionary branching at the degenerate singularity.

Evolutionary branching gives rise to a polymorphism of two substantially different strategies in an initially monomorphic population. The subsequent coevolution of the coexisting strategies can be modelled similarly to the monomorphic populations (Geritz et al., 1998). The fitness of a rare mutant $y$ in the population of resident strategies $x_{p}, \ldots, x_{n}$ is

$$
r_{x_{1}, \ldots, x_{n}}(y)=\rho(y)-\sum_{j=1}^{n} \alpha\left(y-x_{j}\right) N_{j}
$$

(cf. Eq. 1). The fitness gradient

$$
\left.\frac{\partial r_{x_{1}, \ldots, x_{n}}(y)}{\partial y}\right|_{y=x_{i}}=\rho^{\prime}\left(x_{i}\right)-\sum_{j=1}^{n} \alpha^{\prime}\left(x_{i}-x_{j}\right) N_{j}
$$

determines the direction of evolution of the resident strategy $x_{i}$. Notice that in polymorphic populations the shape of function $\alpha$ is significant not only near zero, because mutants of one resident must compete also with other, substantially different residents.

Polymorphic evolutionary singularities are coalitions of resident strategies where the fitness gradient of each strategy is zero. Unfortunately, a general condition for convergence stability is not straightforward in polymorphic populations (Matessi and Di Pascuale, 1996). If the population arrives near the singular coalition, a particular resident strategy $x_{i}$ can undergo evolutionary branching if it is evolutionarily unstable, i.e., if

$$
\left.\frac{\partial^{2} r_{x_{1}, \ldots, x_{n}}(y)}{\partial y^{2}}\right|_{y=x_{i}}=\rho^{\prime \prime}\left(x_{i}\right)-\sum_{j=1}^{n} \alpha^{\prime \prime}\left(x_{i}-x_{j}\right) N_{j}
$$

is positive. This condition is necessary; however, it is sufficient only if all the other strategies of the singular coalition are evolutionarily stable, and hence remain at the singularity. If other strategies also lack evolutionary stability, and they evolve with different speed, then the fastest of them can undergo branching while the others may lag behind. As branching in one strategy generally changes the frequency-dependent fitness function (Eq. 9), the slower evolving strategies may no longer be near a branching 
point, i.e., they may have missed the opportunity for branching. Faster evolution of a strategy is possible if it is present in greater number (since the speed of evolution is limited by the appearance of new mutants, and the number of mutants is proportional to the number of individuals), or if the rate or size of mutations depends on the trait value.

Polymorphic populations with asymmetric competition may have very rich adaptive dynamics, a full exploration of which is beyond the scope of this paper. The following examples illustrate the possibility of evolution to a stable polymorphism, repeated evolutionary branching, "missed" branching, and extinction following evolutionary branching.

\section{Examples}

The following three examples assume different functional forms for the intrinsic growth rate $\rho(x)$. The competitive coefficient function $\alpha(y-x)$ remains unspecified; in the numerical analyses of polymorphic populations, however, I use Eq. 2 for $\alpha(y-x)$ with different values of $v$. If $v<1$ then $\alpha$ is convex, if $v>1$ then $\alpha$ is concave, and if $v=1$ then $\alpha$ has an inflection point at $y-x=0$.

\section{Example 1: Linear $\rho(x)$}

If the intrinsic growth rate is a linearly decreasing function of the trait value,

$$
\rho(x)=\beta-b x
$$

then $\left.\frac{\partial r_{x}(y)}{\partial y}\right|_{y=x=x^{*}}=0$ can be solved explicitly for the monomorphic singular strategy

$$
x^{*}=\frac{\beta}{b}+\frac{\alpha(0)}{\alpha^{\prime}(0)}
$$

The singularity is always convergence stable; evolutionary branching occurs if $\alpha$ is concave near zero (Table 1 ).

Coevolution following evolutionary branching can be analyzed numerically using Eqs. 10 and 11. The results are shown in Fig. 2a for a dimorphic population where $\alpha$ is given by Eq. 2. For each pair of coexisting strategies, the direction of evolution of $x_{1}$ and $x_{2}$ can be determined from Eq. 10: In Fig. 2a, these are indicated by horizontal and vertical arrows, respectively, within the set of protected dimorphisms. Directional evolution of one or the other resident strategy ceases at the so-called isoclines. The intersection of the isoclines thus corresponds to the dimorphic evolutionary singularity. 
(a)

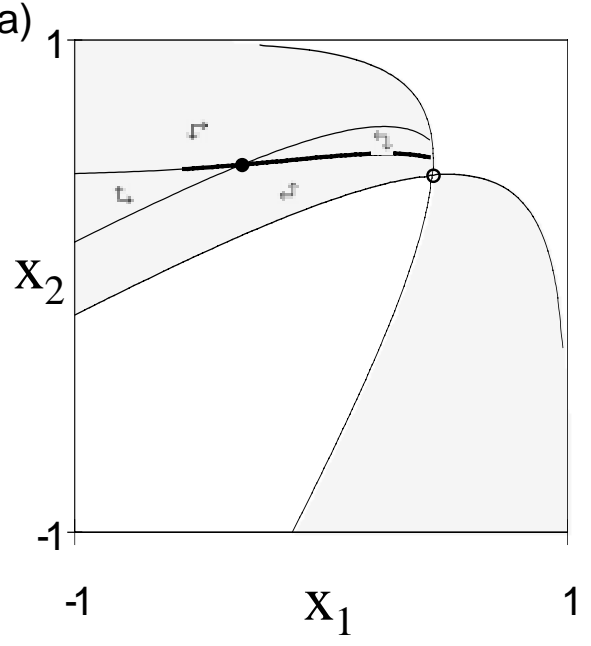

(b)

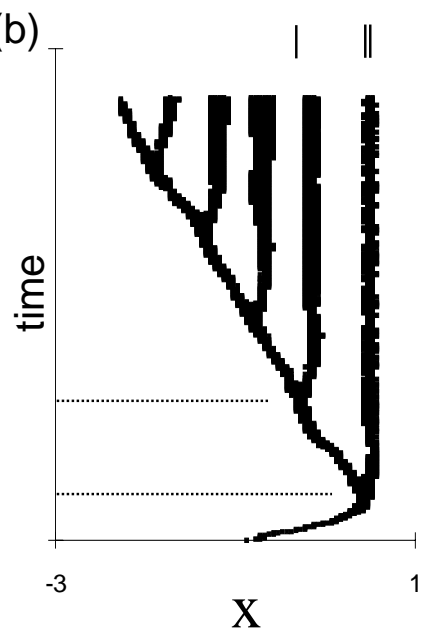

Fig. 2. (a) Isocline plot with linear $\rho(x)$. Strategy pairs inside the shaded area can form a protected dimorphism. The direction of evolution of $x_{1}\left(x_{2}\right)$ is shown by horizontal (vertical) arrows as determined from Eq. 10. Thick isoclines are evolutionarily stable (Eq. 11 is negative); thin isoclines are evolutionarily unstable (Eq. 11 is positive). The open circle indicates the branching point $\left(x_{1}=x_{2}=x^{*}=0.45\right)$, the filled circle corresponds to the dimorphic singular coalition, $\left(x_{1}^{*}, x_{2}^{*}\right)=(-0.32,0.49)$. At the singular coalition, $x_{1}$ (i.e., the smaller strategy) is evolutionarily unstable, $x_{2}$ (the larger strategy) is evolutionarily stable. (b) Simulated evolutionary tree. Starting with a monomorphic population, evolution first converges to $x^{*}=0.45$ where it undergoes evolutionary branching; the two branches evolve to $\left(x_{1}^{*}, x_{2}^{*}\right)=(-0.32,0.49)$, where the smaller strategy undergoes branching again. Subsequent evolution leads to increasing levels of polymorphism by repeated branching. The position of the branching point and the dimorphic singular coalition, as read from the isocline plot, are shown by the vertical bars above the tree; horizontal dotted lines indicate when the population is at the monomorphic and dimorphic singularities. Parameter values: $\beta=1, b=1, c=2, v=1.2$, $k=4$.

The expected course of evolution of the dimorphic population can be read from the isocline plot in Fig. 2a as follows. For convenience, I assume that the smaller strategy of the coalition is denoted by $x_{1}$ and the larger one is $x_{2}$ (that is, I consider the upper half of the plot above the $x_{2}=x_{1}$ diagonal). At the beginning of evolutionary branching, the population contains two strategies both near $x^{*}$, which undergo disruptive coevolution, i.e., $x_{1}$ decreases and $x_{2}$ increases. Evolution soon arrives at the $x_{2}$-isocline, and proceeds along this isocline towards the singular coalition $\left(x_{1}^{*}, x_{2}^{*}\right)$. Since the $x_{2}$-isocline is nearly horizontal, the larger strategy will stay approximately constant while the smaller strategy evolves towards even smaller trait values. In the singular coalition, the large strategy is evolutionarily stable, but the small strategy is evolutionarily unstable (as determined from Eq. 11 and indicated by the thickness of the isoclines). The small strategy thus undergoes secondary branching, giving rise to a trimorphic population. Subsequent evolution can be analyzed similarly to the dimorphic case; however, I do not pursue the numerical analysis further because the results cannot be visualized. 
With linear $\rho(x)$, a strategy $x_{i}$ in the $n$-morphic singular coalition lacks evolutionary stability if $-\sum_{j=1}^{n} \alpha^{\prime \prime}\left(x_{i}-x_{j}\right) N_{j}$ is positive (cf. Eq. 11). Notice that this condition is always satisfied for the smallest strategy of the coalition, provided that $\alpha$ is concave-convex and $\alpha^{\prime \prime}(0)<0$. The first branching is thus followed by a series of further evolutionary branching events; the series of repeated branching is interrupted only if the smallest possible trait value is reached. Depending on the model parameters, some of the larger strategies can undergo further branching as well.

I also performed direct simulations of the evolutionary process in order to confirm the model predictions, and to illustrate the evolutionary dynamics of three and more coexisting strategies (Fig. 2b). In the simulations, I iterated the population dynamics according to Eq. 1, starting with a monomorphic population. Mutant strategies were generated by small deviations from strategies already present, and were introduced at a low initial frequency. By the iteration of the population dynamics some strategies grew in number, others declined. If the frequency of a strategy dropped below a low threshold, the strategy was considered extinct and was removed from the iteration. The evolutionary tree shows the strategies present at various times during the simulation. The simulations were not mutation-limited, i.e., mutants appeared before the previous mutants reached their equilibrium density or went extinct. As a consequence, there was always some variation within a branch of the evolutionary tree. This variation, however, did not confound the model predictions.

\section{Example 2: Gaussian $\rho(x)$}

Stabilizing selection on the trait can be modelled by assuming that the intrinsic growth rate is a Gaussian function of the trait value,

$$
\rho(x)=a \exp \left(-\frac{(x-m)^{2}}{2 \sigma^{2}}\right)
$$

In this case, there is a single monomorphic singularity at

$$
x^{*}=m-\frac{\alpha^{\prime}(0)}{\alpha(0)} \sigma^{2}
$$

that is larger than the optimal trait value in a competitive-free environment $(m)$ because $\alpha^{\prime}(0)$ is negative. The singular strategy is always convergence stable (Inequality 5 reduces to $\left.-\rho\left(x^{*}\right) / \sigma^{2}<0\right)$. The monomorphic population undergoes evolutionary branching if

$$
\left.\frac{\partial^{2} r_{x}(y)}{\partial y^{2}}\right|_{y=x=x^{*}}=\rho\left(x^{*}\right)\left[\left(\frac{\alpha^{\prime}(0)}{\alpha(0)}\right)^{2}-\frac{1}{\sigma^{2}}-\frac{\alpha^{\prime \prime}(0)}{\alpha(0)}\right]>0
$$


(a)

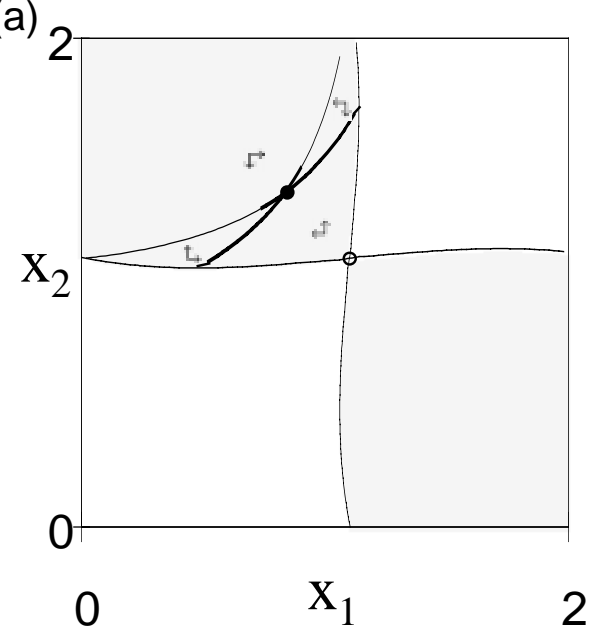

(b)

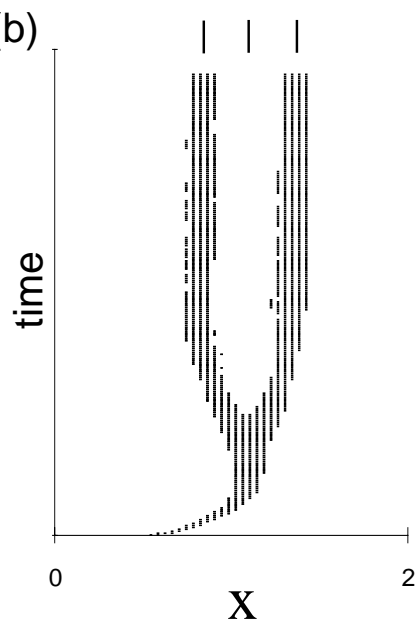

Fig. 3. (a) Isocline plot with Gaussian $\rho(x)$. (b) Simulated evolutionary tree. The monomorphic population has a branching point at $x_{1}=x_{2}=x^{*}=1.1$; there is an evolutionarily stable dimorphic coalition at $\left(x_{1}^{*}, x_{2}^{*}\right)=(0.84,1.37)$. Notations as in Fig. 2. Parameter values: $a=1, m=0, \sigma=1, c=2, v=1, k=2.2$.

Evolutionary branching is hence promoted if (i) $\left|\frac{\alpha^{\prime}(0)}{\alpha(0)}\right|$ is large, i.e., there is strong asymmetry in competition, (ii) $\sigma^{2}$ is large, i.e., stabilizing selection is weak, and (iii) $\alpha$ is concave at zero. Strong asymmetry and weak stabilizing selection result in a large $x^{*}$ (cf. Eq. 15), such that the Gaussian function $\rho(x)$ is convex at $x^{*}$, which favors branching (Table 1).

Following evolutionary branching, the population may reach an evolutionarily stable coalition of two strategies provided that the competitive asymmetry is sufficiently weak, i.e., $k$ is sufficiently small in Eq. 2 (Fig. 3). With stronger asymmetry, however, further evolutionary branching occurs. The isocline plot in Fig. 4a shows an example where both strategies of the dimorphic singular coalition lack evolutionary stability. The population density of the smaller strategy is higher, hence it produces more mutants and evolves faster. Once the smaller strategy has undergone branching, the fitness function (Eq. 9) slightly changes such that the singularity shifts away from the present trait value of the larger strategy. Whether or not the larger strategy will still undergo branching depends on the size of mutations relative to the magnitude of this shift: If mutations are not very small, then the larger strategy can still branch, but if mutations are of sufficiently small phenotypic effect, then the opportunity for evolutionary branching in the larger strategy is missed. Fig. 4b illustrates a simulation with missed branching; an analogous simulation with somewhat larger mutations led to branching in both strategies of the dimorphic coalition. 

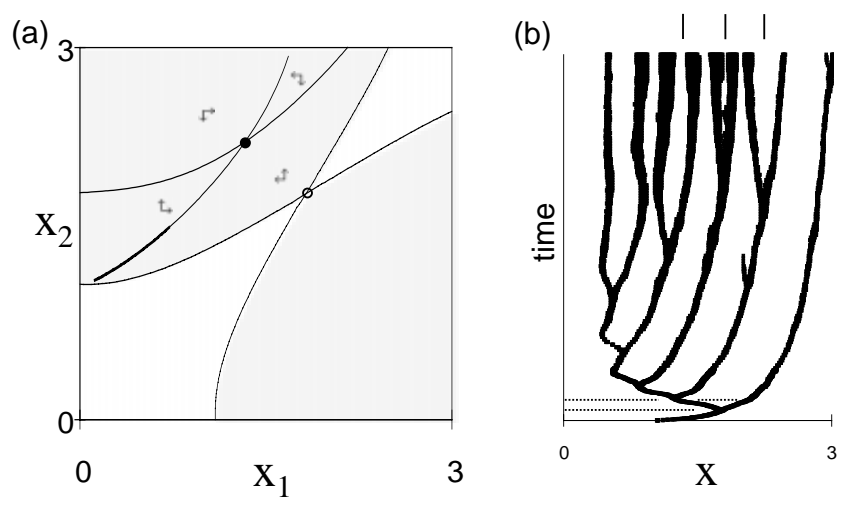

Fig. 4. (a) Isocline plot with Gaussian $\rho(x)$. (b) Simulated evolutionary tree. The monomorphic population has a branching point at $x_{1}=x_{2}=x^{*}=1.81$. In the dimorphic singular coalition $\left(\left(x_{1}^{*}, x_{2}^{*}\right)=(1.33,2.24)\right)$, both strategies lack evolutionary stability; in the simulation, however, the larger strategy at the two-strategy stage misses evolutionary branching. Notations as in Fig. 2. Parameter values: $a=1, m=0, \sigma=1, c=2$, $v=1.2, k=4$.

Repeated evolutionary branching can lead to high levels of polymorphism (Fig. $4 b)$. Unlike in the case of linear $\rho(x)$, the coexisting strategies are confined in range such that all strategies are larger than $m$, the optimal trait value in a competition-free environment: Strategies below $m$ are disadvantageous with respect to both stabilizing selection and asymmetric competition, and therefore would experience strong selection towards larger trait values.

\section{Example 3: Convex $\rho(x)$}

If the intrinsic growth rate is a convex function of the trait value, then a monomorphic population may have multiple evolutionary singularities. Here I assume the monotonicly decreasing, convex function

$$
\rho(x)=-a-b\left(x-\sqrt{x^{2}+d}\right)
$$

with non-negative $a$ and positive $b$ and $d$. For large negative values, this function is approximately linear with slope $-2 b$. As $x$ increases, the function becomes less steep; for large positive values of $x$, it asymptotically converges to $-a$.

If $a=0$, i.e., if even very large strategies have positive growth rates in a competition-free environment, then there are two monomorphic evolutionary singularities at

$$
x_{1,2}^{*}= \pm \sqrt{\left(\frac{\alpha(0)}{\alpha^{\prime}(0)}\right)^{2}-d}
$$

The larger singular strategy is always convergence unstable, hence an initial population above this singularity undergoes runaway evolution towards large trait values. The smaller singularity is a branching point unless $\alpha$ is too convex (Table 1). If competitive 
asymmetry is strong such that $\left(\frac{\alpha(0)}{\alpha^{\prime}(0)}\right)^{2}<d$, then there is no evolutionarily singular strategy: Runaway evolution leads to ever increasing trait values unless the strategy set is constrained. As the trait value increases, the equilibrium population density, $N(x)=\rho(x) / \alpha(0)$, decreases: the population evolves towards extinction (Matsuda and Abrams, 1994).

If $a>0$, then $\rho(x)$ is negative for large trait values, and therefore runaway evolution is not possible. The monomorphic evolutionary singularities are the roots of a third-degree polynomial; the largest one is always convergence stable. Fig. 5 shows the singular trait values as a function of competitive asymmetry. If $\alpha$ is convex near zero (Fig. 5a), then there may be one or two convergence stable ESSs; if $\alpha$ is concave (Fig. 5b), then all convergence stable singularities are branching points (cf. Table 1).

(a)
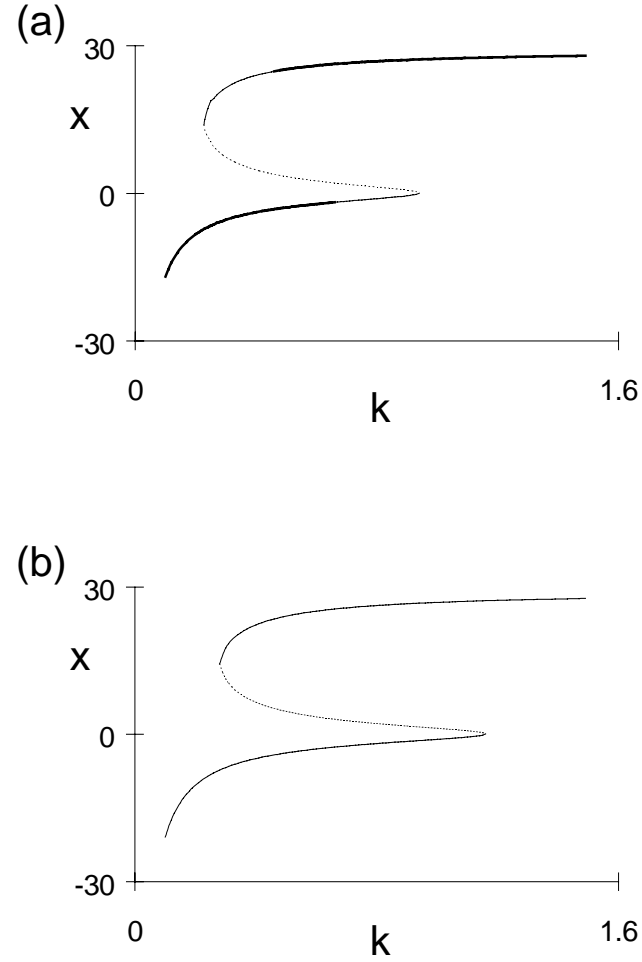

Fig. 5. The monomorphic evolutionary singularities for convex $\rho(x)$ as given by Eq. 17. $\alpha$ is specified by Eq. 2 ; increasing $k$ corresponds to increasingly asymmetric competition. Thick lines denote evolutionarily stable strategies, thin lines are branching points, and dotted lines are evolutionary repellors. (a) $\alpha$ is convex near zero $(v=0.7)$; (b) $\alpha$ is concave near zero $(v=1.1)$. Parameter values: $a=0.6, b=10, d=3.5, c=2$.

Evolutionary branching gives rise to protected polymorphism. It does not guarantee, however, that the polymorphism is persistent on an evolutionary timescale: Coevolution 
of the coexisting strategies can lead to extinction, and thus the population can fall back to a lower level of polymorphism. Such an evolutionary scenario is illustrated in Fig. 6. There are two convergence stable monomorphic evolutionary singularities, an ESS and a branching point, with a repellor inbetween. A monomorphic population above the repellor evolves to the branching point and becomes dimorphic. However, there is no dimorphic singularity in the domain of protected dimorphisms in which the population is evolving. Therefore the population eventually leaves the set of protected dimorphisms, and the larger strategy of the dimorphism goes extinct. The remaining monomorphic population is below the repellor and hence evolves to the monomorphic ESS.

(a)

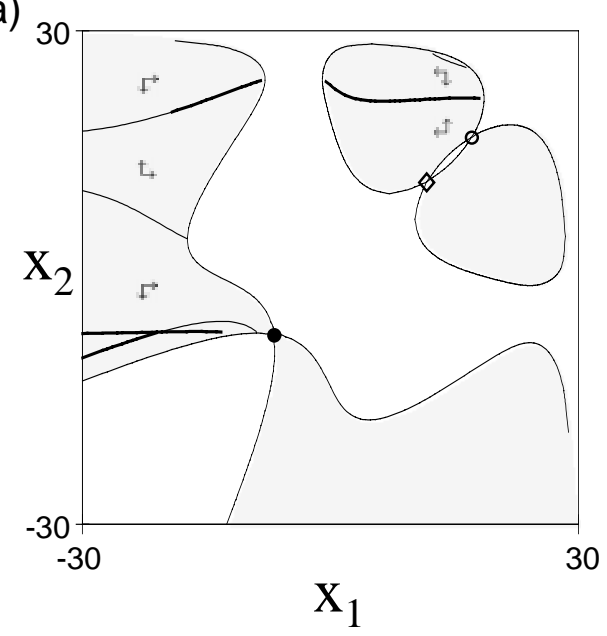

(b)

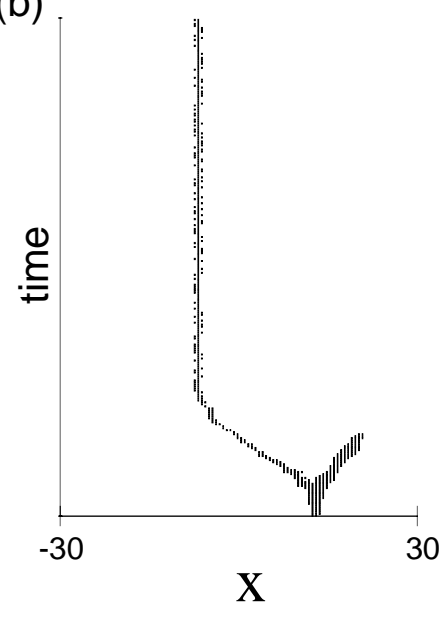

Fig. 6. (a) Isocline plot with convex $\rho(x)$ as given by Eq. 17. The monomorphic population has three singularities, an ESS at $x_{1}=x_{2}=x^{*}=-6.86$ (filled circle), a repellor at $x_{1}=x_{2}=x^{*}=11.37$ (diamond), and a branching point at $x_{1}=x_{2}=x^{*}=17.22$ (open circle); other notations as in Fig. 2. (b) Simulated evolutionary tree. Starting with a monomorphic population above the repellor, the population evolves to the branching point and undergoes evolutionary branching; the dimorphic population, however, leaves the area of protected dimorphism, i.e., the larger strategy goes extinct. The remaining monomorphic population is below the repellor and therefore evolves to the ESS. Parameter values: $a=0.6, b=10, d=3.5, c=2, v=0.7, k=0.24$.

\section{Disc ussion}

In a simple asymmetric competition model based on the Lotka-Volterra equations, I have demonstrated the possibility for evolutionary branching, i.e., the evolution of polymorphism by small mutational steps in an initially monomorphic population. 
Repeated evolutionary branching readily leads to high levels of polymorphism (e.g., Figs. $2 b$ and $4 b)$.

Evolutionary branching is possible only if a large and a small strategy can coexist in a protected dimorphism. A large strategy can invade the population of small individuals by its competitive superiority. A small strategy can, however, also invade the population of large individuals. Though small individuals cannot win a contest against large ones, they have a good chance to avoid any such contest altogether, because the large strategy can maintain only a low population density due to the cost of large trait values. The few contests encountered and lost by small individuals is overcompensated by their greater intrinsic growth rate, i.e., by not bearing the cost of large size.

When runaway evolution proceeds up to large trait values, then even a slightly smaller mutant can invade and coexist with the former resident. This sets the initial stage of evolutionary branching. If the two coexisting strategies experience disruptive selection (which is mathematically equivalent to there being no evolutionarily stable strategy sufficiently similar to them), then divergent coevolution gives rise to two phenotypically distinct branches.

For simplicity, I always started with a monomorphic population and investigated whether it can undergo evolutionary branching. Evolutionary branching, however, may occur more easily in an initially polymorphic population. Such an example is shown in Fig. 7. Here the monomorphic population has an ESS, therefore no polymorphism evolves (Fig. 7a). If, however, the initial population is dimorphic, then the smaller strategy undergoes repeated evolutionary branching whereby a high level of polymorphism can evolve (Fig. 7b). This implies that there may be a threshold level of polymorphism above which higher level polymorphisms can be evolutionarily restored. If some environmental catastrophe were to kill all but two strategies of the rich coalition built up in Fig. 7b, then highly polymorphic coalitions can evolve again provided that the two remaining strategies are able to coexist and evolve to the dimorphic branching point. If only one strategy is left, then it will evolve to the ESS, thus the polymorphism cannot be regained. 
(a)

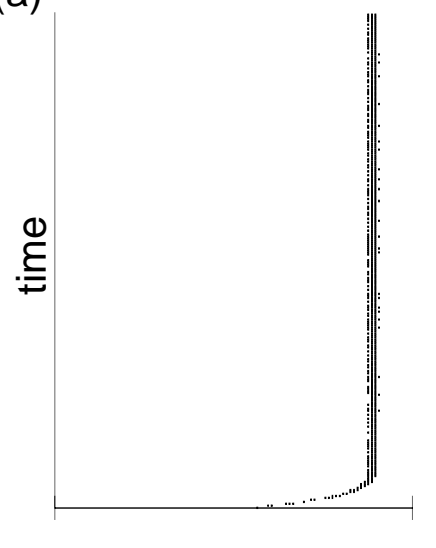

(b)

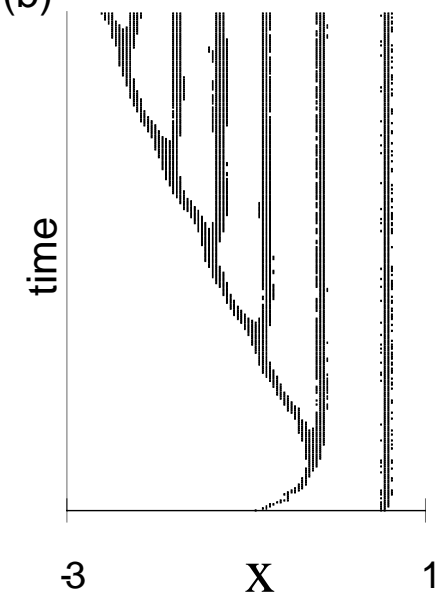

Fig. 7. Simulated evolutionary trees. (a) Starting with a monomorphic population, evolution converges to an ESS. (b) Starting with a dimorphic population, the smaller strategy undergoes repeated evolutionary branching. $\rho(x)$ is linear (Eq. 12), $\alpha$ is given by Eq. 2; parameter values are $\beta=1, b=1, c=2, v=0.8, k=4$.

The Lotka-Volterra type model used in this paper is perhaps the simplest model of asymmetric competition. It is not, however, a mechanistic model, i.e., it is not derived from underlying elementary processes of individual life histories. When trading off ecological realism for simplicity, it is crucial to ask how robust the predictions are with respect to structural modifications of the model.

A short preliminary analysis of the asymmetric competition model proposed by Roughgarden in order to explain taxon cycles (Roughgarden, 1979; Rummel and Roughgarden, 1985; Brown and Vincent, 1987; Taper and Case, 1992) also indicated evolutionary branching (Kisdi, unpubl.). In these models, however, different strategies exploit partially different niches, and niche segregation is known to promote evolutionary branching without competitive asymmetry (Metz et al. 1996).

In a model constructed specifically for the evolution of seed size in plants, the results obtained by Geritz et al. (in press) were strikingly similar to the present study, including evolutionary branching, repeated branching, and extinction. This is surprising because their model was rather different from the simple Lotka-Volterra model used here. Seeds were assumed to disperse randomly into safe-sites, such that the number of seeds per site was variable. Competition was restricted to seeds within the same site. Individuals competed according to a weighted lottery, where an increase in seed size conferred a disproportionate advantage as larger seedlings oppress smaller ones by shading. Larger seeds also had higher precompetitive survival probability; large seeds, however, could be produced in less number given the limited resources provided by a safe site. The fitness function incorporating these ecological details is analytically untractable and much more complicated than Eq. 3. Nevertheless the predicted evolutionary scenarios are similar, which may reflect a fundamental similarity of the evolutionary mechanism operating in the two models: The evolutionary increase of the trait value promoted by competitive asymmetry leads to low population density, where competition is relaxed and smaller mutants can invade. 
It would be premature, however, to conclude that competitive asymmetry facilitates evolutionary branching in general. A reanalysis of four asymmetric competition models used by Abrams and Matsuda (1994) shows that evolutionary branching is possible in only two of them (Kisdi, in prep.). Further research is required in order to identify the critical ecological factors which determine whether asymmetric competition can lead to evolutionary branching.

As most previous models with evolutionary branching (Metz et al. 1996; Doebeli and Ruxton, 1997; Meszéna et al., 1997; Geritz et al., 1998, in press; Geritz and Kisdi, in press; Mathias and Kisdi, in press; Meszéna and Metz, in press), I modelled evolution on a phenotypic level and assumed that strategies breed true (i.e., there is haploid or clonal inheritance). Phenotypic adaptive dynamics of monomorphic populations (termed often as the 'ESS-approach') is largely compatible with quantitative genetic models (Charlesworth, 1990; Iwasa et al., 1991; Taper and Case, 1992; Abrams et al., 1993a,b; Taylor, 1996). In polymorphic populations, however, random mating and recombination re-creates the intermediate phenotypes which are selected against during evolutionary branching, and thereby prevent the emergence of two phenotypically separate branches. Abrams et al. (1993a) therefore argued that the evolution of polygenic traits gets stuck at the branching point, in spite of that the population is at a fitness minimum.

In a one-locus diploid adaptive dynamics model, Kisdi and Geritz (ms) showed that alleles undergo evolutionary branching much like haploid genotypes in the phenotypic model, giving rise to a protected genetic polymorphism of distinctly different alleles. Since intermediate heterozygotes are inferior during evolutionary branching, there is selection for assortative mating which reproductively isolates the branches. Although the evolution of assortative mating due to hybrid inferiority (reinforcement) is highly controversial, there is some empirical evidence which seems to support the possibility (e.g., Coyne and Orr, 1989, 1997; Johannesson et al., 1995; Noor, 1995; Saetre et al., 1997; Rundle and Schluter, 1998). If the population has split up into two reproductively isolated species, further evolution proceeds according to the phenotypic (haploid) adaptive dynamics: The two sibling species diverge from the branching point and become phenotypically distinct.

If the trait under selection determines mate choice, then evolutionary branching immediately results in prezygotic isolation (Templeton, 1981; Rice, 1984; Smith, 1988; Rice and Salt, 1990; Rice and Hostert, 1993; Doebeli, 1996; Galis and Metz, 1998). Body size has a strong influence on mate choice, and disruptive selection on body size is thought to lead to sympatric speciation in several species (Schluter and Nagel, 1995; Nagel and Schluter, 1998). Body size is often a main factor in competitive asymmetry. It is thus reasonable to expect that asymmetric competition, by generating disruptive selection on body size at an evolutionary branching point, may cause sympatric speciation when mating is size-assortative.

If there is no assortative mating based on body size (or on some other primary trait) in the initial population, it may still evolve when the population is at the 
evolutionary branching point. Under disruptive selection on the primary trait, an allele influencing mate choice such that carriers prefer mates with primary trait similar to their own is spread by selection (Maynard Smith, 1966; Seger, 1985; Doebeli, 1996; Doebeli and Dieckmann, in press). Unlike other preferential mating mechanisms, assortative mating based on the primary trait evolves easily because no linkage disequilibrium is needed between the primary trait and the mate choice loci (Felsenstein, 1981). Ensuring reproductive isolation between the branches, assortative mating essentially restores true breeding and makes evolutionary branching of polygenic traits possible. 


\section{References}

Abrams, P. A., and H. Matsuda. 1994. The evolution of traits that determine ability in competitive contests. Evol. Ecol. 8:667-686.

Abrams, P. A., H. Matsuda, and Y. Harada. 1993a. Evolutionarily unstable fitness maxima and stable fitness minima of continuous traits. Evol. Ecol. 7:465-487.

Abrams, P. A., Y. Harada, and H. Matsuda. 1993b. On the relationship between quantitative genetic and ESS models. Evolution 47:982-985.

Alatalo, R. V., and J. Moreno. 1987. Body size, interspecific interactions, and the use of foraging sites in tits (Paridae). Ecology 68:1773-1777.

Begon, M. 1984. Density and individual fitness: Asymmetric competition. Pp. 175-194 in B. Shorrocks, ed. Evolutionary Ecology. Blackwell Science Inc., Oxford.

Begon, M., J. L. Harper, and C. L. Townsend. 1996. Ecology. Blackwell Science Inc., Oxford.

Brown, J. S., and T. L. Vincent. 1987. Coevolution as an evolutionary game. Evolution 41:66-79.

Carroll, S. P., and M. H. Salamon. 1995. Variation in sexual selection on male body size within and between populations of the soapberry bug. Anim. Behav. 50:1463-1474.

Charlesworth, B. 1990. Optimization models, quantitative genetics, and mutation. Evolution 44:520-538.

Clutton-Brock, T. H., and S. D. Albon. 1979. The roaring of red deer and the evolution of honest advertisement. Behaviour 69:145-170.

Clutton-Brock, T. H., S. D. Albon, R. M. Gibson, and F. E. Guinness. 1979. The logical stag: Aspects of fighting in red deer (Cervus elaphus L.). Anim. Behav. 27:211-275.

Connell, J. H. 1983. On the prevalence and relative importance of interspecific competition: Evidence from field experiments. Am. Nat. 122:661-696.

Coyne, J. A., and H. A. Orr. 1989. Patterns of speciation in Drosophila. Evolution 43:362-381.

Coyne, J. A., and H. A. Orr. 1997. "Patterns of speciation in Drosophila," revisited. Evolution 51:295-303.

Christiansen, F. B. 1991. On conditions for evolutionary stability for a continuously varying character. Am. Nat. 138:37-50.

Dawkins, R., and J. R. Krebs. 1979. Arms races between and within species. Proc. R. Soc. Lond. B 205:489-511.

Dickman, C. R. 1988. Body size, prey size, and community structure in insectivorous mammals. Ecology 69:569-580.

Doebeli, M. 1996. A quantitative genetic model for sympatric speciation. J. Evol. Biol. 9:893-909. 
Doebeli, M., and U. Dieckmann. Evolutionary branching with multi-locus genetics. in U. Dieckmann, and J. A. J. Metz, eds. Advances in adaptive dynamics. Cambridge University Press. In press.

Doebeli, M., and G. D. Ruxton. 1997. Evolution of dispersal rates in metapopulation models: Branching and cyclic dynamics in phenotype space. Evolution 51:1730-1741.

Englund, C., F. Johansson, and T. I. Olsson. 1992. Asymmetric competition between different taxa: Poecilid fishes and water striders. Oecologia 92:498-502.

Eshel, I. 1983. Evolutionary and continuous stability. J. theor. Biol. 103:99-111.

Eshel, I., U. Motro, and E. Sansone. 1997. Continuous stability and evolutionary convergence. J. theor. Biol. 185:333-343.

Felsenstein, J. 1981. Scepticism towards Santa Rosalia, or why are there so few kinds of animals?. Evolution 35: 124-138.

Galis, F., and J. A. J. Metz. 1998. Why are there so many cichlid species? TREE 13:1-2.

Geritz, S. A. H. 1995. Evolutionarily stable seed polymorphism and small-scale spatial variation in seedling density. Am. Nat. 146:685-707.

Geritz, S. A. H., and É. Kisdi. Evolutionary branching and sympatric speciation in diploid populations. In: U. Dieckmann, and J. A. J. Metz, eds. Advances in adaptive dynamics. Cambridge University Press. In press.

Geritz, S. A. H., J. A. J. Metz, É. Kisdi, and G. Meszéna. 1997. Dynamics of adaptation and evolutionary branching. Phys. Rev. Letters 78:2024-2027.

Geritz, S. A. H., É. Kisdi, G. Meszéna, and J. A. J. Metz. 1998. Evolutionarily singular strategies and the adaptive growth and branching of the evolutionary tree. Evol. Ecol. 12:35-57.

Geritz, S. A. H., E. van der Meijden, and J. A. J. Metz. Evolutionary dynamics of seed size and seedling competitive ability. In press.

Iwasa, Y., A. Pomiankowski, and S. Nee. 1991. The evolution of costly mate preferences. II. The 'handicap' principle. Evolution 45:1431-1442.

Johannesson, K., E. Rolan-Alvarez, and A. Ekendahl. 1995. Incipient reproductive isolation between two sympatric morphs of the intertidal snail Lyttorina saxatilis. Evolution 49:1180-1190.

Kisdi, É., and S. A. H. Geritz. Adaptive dynamics in allele space: Evolution of genetic polymorphism by small mutations in a heterogeneous environment. manuscript

Law, R., P. Marrow, and U. Dieckmann. 1997. On evolution under asymmetric competition. Evol. Ecol. 11:485-501.

Lawton, J. H., and M. P. Hassell. 1981. Asymmetrical competition in insects. Nature 289:793-795.

Lomnicki, A. 1989. Avoiding chaos. TREE 4:239.

Luiselli, L. 1996. Individual success in mating balls of the grass snake, Natrix natrix: Size is important. Journal of Zoology (London) 239:731-740. 
Matessi, C., and C. Di Pasquale. 1996. Long-term evolution of multilocus traits. J. Math. Biol. 34:613-653.

Mathias, A., and É. Kisdi. Evolutionary branching and coexistence of germination strategies. in U. Dieckmann, and J. A. J. Metz, eds. Advances in adaptive dynamics. Cambridge University Press. In press.

Matsuda H. and P. A. Abrams. 1994. Runaway evolution to self-extinction under asymmetrical competition. Evolution 48:1764-1772.

Maynard Smith, J. 1966. Sympatric speciation. Am. Nat. 100:637-650.

Maynard Smith, J. 1982. Evolution and the theory of games. Cambridge University Press, Cambridge.

Maynard Smith, J., and R. L. Brown. 1986. Competition and body size. Theor. Pop. Biol. 30:166-179.

Meszéna, G., I. Czibula, and S. A. H. Geritz. 1997. Adaptive dynamics in a 2-patch environment: A toy model for allopatric and parapatric speciation. J. Biol. Syst. 5:265-284.

Meszéna, G., and J. A. J. Metz. The role of effective environmental dimensionality. in U. Dieckmann, and J. A. J. Metz, eds. Advances in adaptive dynamics. Cambridge University Press. In press.

Metz, J. A. J., R. M. Nisbet, S. A. H. Geritz. 1992. How should we define 'fitness' for general ecological scenarios? TREE 7:198-202.

Metz, J. A. J., S. A. H. Geritz, G. Meszéna, F. J. A. Jacobs, and J. S. van Heerwaarden. 1996. Adaptive dynamics, a geometrical study of the consequences of nearly faithful reproduction. Pp. 183-231 in S. J. van Strien, and S. M. Verduyn Lunel, eds. Stochastic and spatial structures of dynamical systems. North Holland, Amsterdam, The Netherlands.

Mitani, J. C., J. Gros Louis, and A. F. Richards. 1996. Sexual dimorphism, the operational sex ratio, and the intensity of male competition in polygynous primates. Am. Nat. 147:966-980.

Nagel, L., and D. Schluter. 1998. Body size, natural selection, and speciation in sticklebacks. Evolution 52: 209-218.

Noor, M. A. 1995. Speciation driven by natural selection in Drosophila. Nature 375:674-675.

Parker, G. A. 1983. Arms races in evolution - an ESS to the opponent-independent costs game. J. theor. Biol. 101:619-648.

Persson, L. 1985. Asymmetric competition: Are larger animals competitively superior? Am. Nat. 126:261-266.

Rice, W. R. 1984. Disruptive selection on habitat preference and the evolution of reproductive isolation: A simulation study. Evolution 38:1251-1260.

Rice, W. R., and G. W. Salt. 1990. The evolution of reproductive isolation as a correlated character under sympatric conditions: Experimental evidence. Evolution 44:1140-1152. 
Rice, W. R., and E. E. Hostert. 1993. Laboratory experiments on speciation: What have we learned in 40 years? Evolution 47:1637-1653.

Roberts, S. C. 1996. The evolution of hornedness in female ruminants. Behaviour 133:399-442.

Roughgarden, J. 1979. Theory of population genetics and evolutionary ecology: An introduction. MacMillan, New York.

Rummel J. D., and J. Roughgarden. 1985. A theory of faunal buildup for competition communities. Evolution 39:1009-1033.

Rundle, H. D., and D. Schluter. 1998. Reinforcement of stickleback mate preferences: Sympatry breeds contempt. Evolution 52:200-208.

Saetre, G. P., T. Moum, S. Bures, M. Kral, M. Adamjan, and J. Moreno. 1997. A sexually selected character displacement in flycatchers reinforces premating isolation. Nature 387:589-592.

Schluter, D., and L. M. Nagel. 1995. Parallel speciation by natural selection. Am. Nat. 146:292-301.

Schoener, T. W. 1983. Field experiments on interspecific competition. Am. Nat. 122:240-285.

Seger, J. 1985. Intraspecifc resource competition as a cause of sympatric speciation. Pp. 43-53 in P. J. Greenwood, P. H. Harvey, and M. Slatkin, eds. Evolution. Essays in honour of John Maynard Smith. Cambridge University Press, Cambridge.

Simmons, R. E., and L. Scheepers. 1996. Winning by a neck: Sexual selection in the evolution of giraffe. Am. Nat. 148:771-786.

Smith, D. C. 1988. Heritable difference of Rhagoletis pomonella host races by seasonal asynchrony. Nature 336:66-67.

Taper, M. L., and T. J. Case. 1992. Models of character displacement and the theoretical robustness of taxon cycles. Evolution 46:317-333.

Taylor, P. D. 1989. Evolutionary stability in one-parameter models under weak selection. Theor. Pop. Biol. 36:125-143.

Taylor, P. D. 1996. The selection differential in quantitative genetics and ESS models. Evolution 50:2106-2110.

Templeton, A. R. 1981. Mechanisms of speciation - a population genetic approach. Ann. Rev. Ecol. Syst. 12:23-48.

Thompson, P., and B. J. Fox. 1993. Asymmetric competition in Australian heathland rodents: A reciprocal removal experiment demonstrating the influence of size-class structure. Oikos 67:264-278.

Weiner, J. 1986. How competition for light and nutrients affects size variability in Ipomea tricolor populations. Ecology 67:1425-1427.

Weiner, J. 1990. Asymmetric competition in plant populations. TREE 5:360-364.

Weiner, J., and S. Thomas. 1986. Size variability and competition in plant monocultures. Oikos 47:211-222. 